\title{
Principal Component Filter Bank for Band Partitioned Sidelobe Cancellation
}

\author{
Peter G. Vouras, Johns Hopkins University \\ Trac D. Tran, Johns Hopkins University
}

Key Words: principal component filter banks, band partitioned sidelobe cancellation

\section{SUMMARY \& CONCLUSIONS}

Principal component filter banks (PCFBs) have been shown to be optimal, if they exist, for a variety of signal processing applications. Ideally, the filters in PCFBs are the eigenvectors of the spectral density matrix of the input random process and therefore depend on the statistics of the input random process. This paper investigates the application of PCFBs to the problem of band partitioned sidelobe cancellation for a two channel canceler. In this context, the filters are the eigenvectors of the cross-spectral density matrix. The ideal filters have an infinite impulse response and are not realizable. Therefore, they must be approximated. Several algorithms are available, but in this paper, the PCFB filters were approximated using a simple, although suboptimal, window method. The cancellation performance of the PCFB was compared to the performance of a time domain GramSchmidt canceler, and band partitioned cancelers utilizing a Dyadic Filter Bank, a Wavelet Packet Filter Bank (WPFB), a Cosine Modulated Filter Bank (CMFB), and a maximally decimated Discrete Fourier Transform Filter Bank (DFTFB). The performance of the approximated PCFB was found to be better than the time domain and dyadic cancelers, but not as good as the DFT, wavelet packet, and cosine modulated cancelers. This shortfall is attributed to the approximation of the ideal PCFB filters.

\section{INTRODUCTION}

A classic system design for removing jamming interference is the Coherent Sidelobe Canceler (CSLC) shown in Figure 1 with two input channels. Both input channels are assumed to be zero mean, Wide Sense Stationary random processes. The primary channel, $y[n]$, in the case of a phased array, is the output of the beamforming network that sums together all the signals from the array elements. The auxiliary or reference channel, $x[n]$, is the signal from one designated array element, or some other auxiliary antenna, that is used to sample the spatial environment. Typically, the gain of the auxiliary antenna is chosen to be higher than the sidelobe level of the array pattern. When a jammer is illuminating the array from some angular offset, the interference will be present in the main channel and ideally a scaled replica of the same interference will be present in the auxiliary channel.

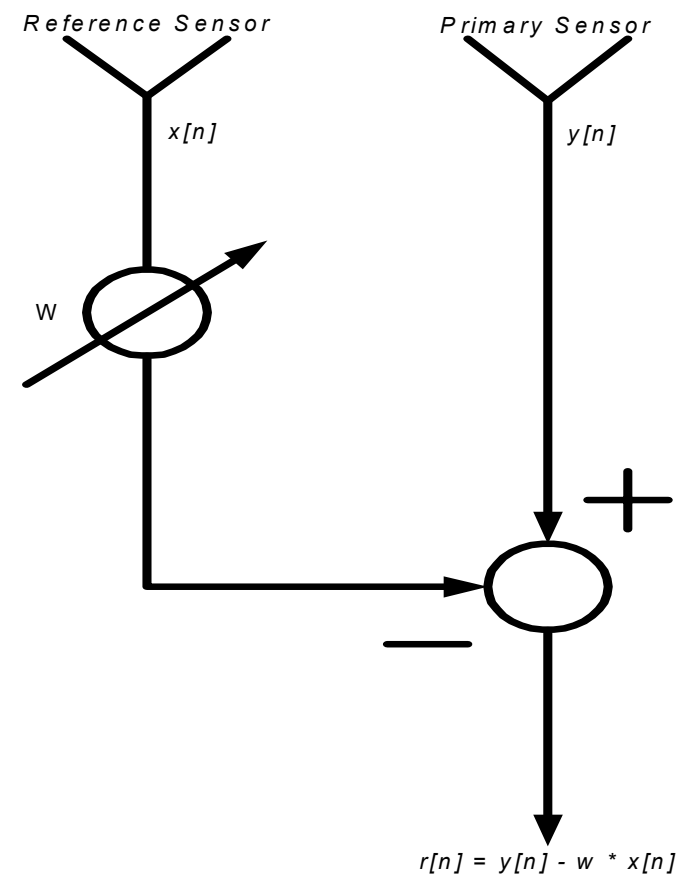

Figure 1. Coherent Sidelobe Canceler

The output of the CSLC is a residue signal that is formed by subtracting the auxiliary channel signal, scaled by some scalar, $w$, from the main channel signal. The objective of the CSLC is to minimize the mean square value of the array output, or $E\left[|r[n]|^{2}\right]$. The solution to this problem, and the optimum weight, $w_{*}$, is the Wiener solution given by,

$$
w_{*}=\frac{E\left[x^{*}[n] y[n]\right]}{E\left[|x[n]|^{2}\right]} .
$$

CSLC performance is usually measured by the cancellation ratio, defined as the output residue power divided by the input main channel power. Experience suggests that the performance of the CSLC is best when the magnitude of the correlation between the auxiliary channel signal and the main channel signal is high. For example, if an exact replica of the interference is available in the auxiliary channel, then the CSLC will completely remove the interference from the main channel and the residue will be thermal noise. In a practical system however, there may be gain and/or phase mismatches between the auxiliary and main channels that 
reduce the channel correlation and thereby limit canceler performance. Also the dispersion of the incident jamming caused by traversing the width of the array will be present in the main channel signal but not in the auxiliary channel signal, and will further limit canceler performance.

\section{FILTER BANKS}

For wideband radar systems the computed canceler weight should be a function of frequency for better performance. By partitioning the received spectrum into contiguous subbands and computing independent weights to be applied to the data in each subband, it is possible to achieve greater cancellation ratios than by simply applying the same weight across all the subbands. A lot of current research has focused on the use of filter banks in adaptive sidelobe cancellation. Examples of this approach are described in References [1] and [2]. The general theory of filter banks is described in Reference [3]. Figure 2 illustrates a maximally decimated filter bank with $M$ channels. Maximally decimated refers to the fact that the decimation factor, $M$, is equal to the number of channels. Maximally decimated filter banks achieve the maximum possible computational efficiency, because the subband signals have the lowest possible sampling rate. It is also possible to have a filter bank with $Q$ channels, where $Q<M$. For the filter bank shown, the input signal is split and convolved with an analysis filter $h[n]$ in each channel. Then the output is decimated by a factor of $M$ and subband processing is performed on each channel independently. The subband processing in this case is adaptive sidelobe cancellation. After the subband processing is complete, the output from each channel is expanded by a factor of $M$ and convolved with a synthesis filter $f[n]$. The filter outputs are then summed together to yield the final output, $\hat{x}[n]$.

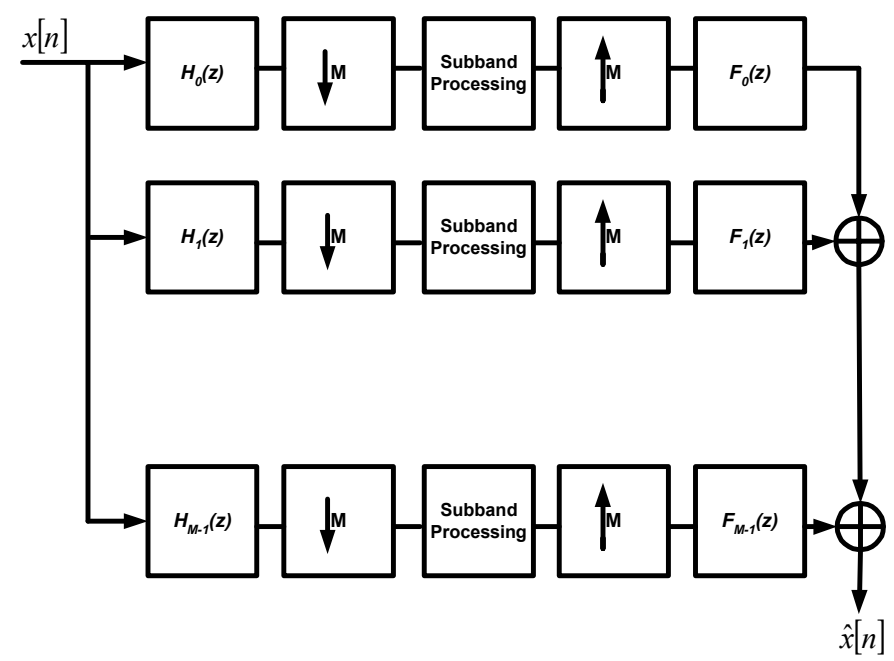

Figure 2. Maximally Decimated Filter Bank

In the absence of subband processing the output signal $\hat{x}[n]$ is expected to match the input signal, $x[n]$, in which case the filter bank is said to achieve perfect reconstruction. A filter bank implementation equivalent to Figure 2 that places the decimation operation before filtering operations and is therefore computationally more efficient, is the polyphase structure shown in Figure 3.

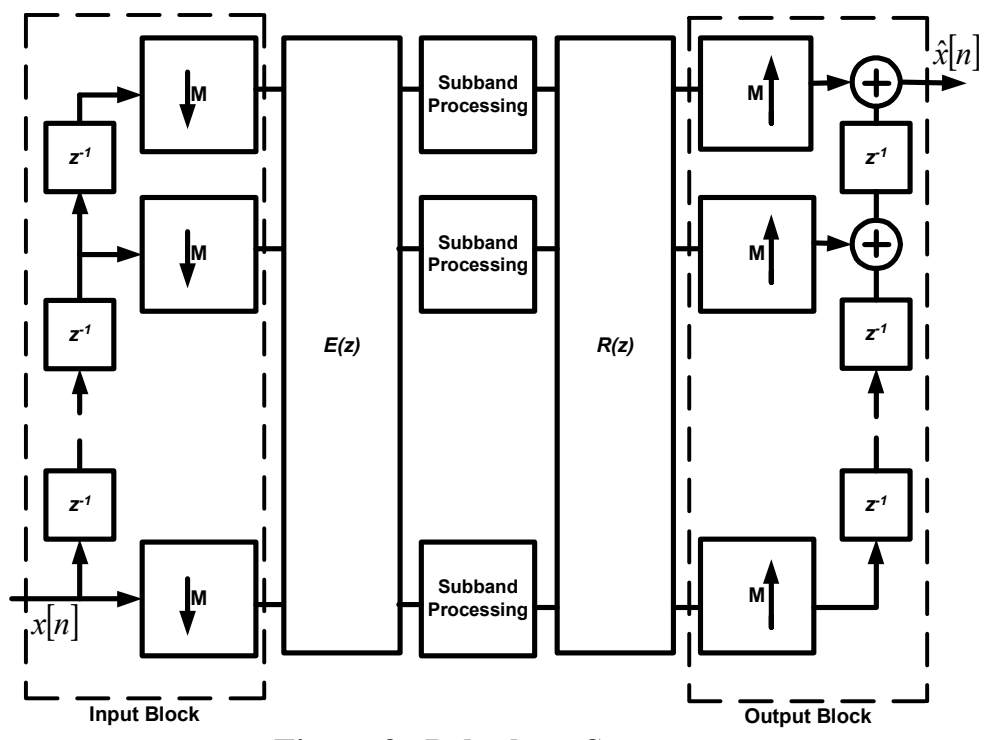

Figure 3. Polyphase Structure

Each analysis filter $H_{k}(z)$ with impulse response $h(n)$ in Figure 2 can be expressed in terms of its polyphase components as,

$$
H_{k}(z)=\sum_{l=0}^{M-1} z^{-l} E_{k l}\left(z^{M}\right), \quad k=0, \ldots, M-1
$$

where

$$
e_{l}(n)=h(l+M n), \quad 0 \leq l \leq M-1
$$

and

$$
E_{l}(z)=\sum_{n=-\infty}^{\infty} e_{l}(n) z^{-n}
$$

Similarly, each synthesis filter can be expressed as

$$
F_{k}(z)=\sum_{l=0}^{M-1} z^{-(M-1-l)} R_{l k}\left(z^{M}\right), \quad k=0, \ldots, M-1 .
$$

Now the $M x M$ polyphase component matrices $E(z)$ and $R(z)$ in Figure 3 can be defined as,

$$
E(z)=\left[E_{k l}(z)\right], \quad R(z)=\left[R_{l k}(z)\right] .
$$

\section{PRINCIPAL COMPONENT FILTER BANKS}

A filter bank that is optimal for subband sidelobe cancellation should maximize the cancellation ratio between the filter bank output and the input compared to all other filter banks. Assume $\vec{x}[n]$ and $\vec{y}[n]$ are two filter bank vector inputs and that a two port CSLC, as shown in Figure 1, is used to cancel the interference within each subband. The output residue from each subband canceler is recombined in the synthesis section of the filter bank to yield a final output residue that is then available for further processing, such as pulse compression.

The correlation between the auxiliary and the main channel signals greatly affects the performance of the sidelobe canceler. One would expect the same observation to hold if 
independent cancelers operate on each of the subband signals, $\vec{x}_{i}[n]$ and $\vec{y}_{i}[n]$ of the filter bank. In other words, for optimum cancellation performance, the magnitude of the cross-correlation sequence $E\left[x_{i}^{*}[n] y_{i}[n]\right]$ should be as large as possible for each filter bank channel.

The main channel signal $x[n]$ consists of two parts, thermal noise which is not correlated with the auxiliary channel signal, and jamming interference which will be correlated to some extent. Thus, represent $x[n]$ and $y[n]$ as,

$$
\begin{gathered}
x[n]=\left\{x \mid E\left[x^{*} y\right]=0\right\}+\left\{x \mid E\left[x^{*} y\right] \neq 0\right\} \\
y[n]=\left\{y \mid E\left[x^{*} y\right]=0\right\}+\left\{y \mid E\left[x^{*} y\right] \neq 0\right\} .
\end{gathered}
$$

Define the signal reconstruction error at the output of the filter bank as,

$$
\begin{aligned}
& e_{y}[n]=y[n]-\hat{y}[n], \\
& e_{x}[n]=x[n]-\hat{x}[n]
\end{aligned}
$$

and define the objective cost function,

$$
J=E\left[e_{x}^{*}[n] e_{y}[n]\right] .
$$

Next, consider the case where,

$$
\begin{aligned}
& \hat{y}[n]=\left\{y \mid E\left[x^{*}[n] y[n]\right]=0\right\} \\
& \hat{x}[n]=\left\{x \mid E\left[x^{*}[n] y[n]\right]=0\right\} .
\end{aligned}
$$

In this case, $J \neq 0$ and $E\left[\hat{x}^{*}[n] \hat{y}[n]\right]=0$, which is not good for CSLC performance. On the other hand, if

$$
\begin{aligned}
& \hat{y}[n]=\left\{y \mid E\left[x^{*}[n] y[n]\right] \neq 0\right\} \\
& \hat{x}[n]=\left\{x \mid E\left[x^{*}[n] y[n]\right] \neq 0\right\},
\end{aligned}
$$

then $J=0$ and $\left|E\left[\hat{x}^{*}[n] \hat{y}[n]\right]\right|>0$ which is good for canceler performance. This argument suggests that to maximize CSLC performance, the analysis and synthesis filters of the filter bank should solve the optimization problem,

$$
\min |\hat{J}|=\left|\lim _{N \rightarrow \infty} \frac{1}{2 N+1} \sum_{n=-N}^{N} E\left[(\vec{x}[n]-\hat{\vec{x}}[n])^{*}(\vec{y}[n]-\hat{\vec{y}}[n])\right]\right|
$$

where

$$
\vec{x}[n]=[x(n M) x(n M-1) \cdots x(n M-M+1)]^{T}
$$

and the vectors $\hat{\bar{x}}[n], \vec{y}[n], \hat{\bar{y}}[n]$ are similarly defined.

The form of this cost function is the same as the cost function considered in Reference [4]. In fact, if the crosscovariance sequence $r_{x y}[\tau]=E\left[x(n+\tau)^{*} y(n)\right]$ is assumed to be Hermitian and positive definite then by proceeding in a manner analogous to the derivation in Reference [4], it can be shown that if the analysis and the synthesis filters of the filter bank correspond to the eigenvectors of the cross-spectral density matrix,

$$
\bar{S}_{X Y}(\omega)=\sum_{\tau=-\infty}^{\infty} \bar{R}_{X Y}(\tau) e^{-j \omega \tau},
$$

where

$$
\bar{R}_{X Y}(\tau)=\lim _{N \rightarrow \infty} \frac{1}{2 N+1} \sum_{n=-N}^{N} R_{X Y}(n ; \tau)
$$

is the time-averaged cross-covariance matrix, then the cost function $|\hat{J}|$ is equal to zero. Then the filter bank is optimal in the sense that the vector of subband cross-correlations, $\left[E\left[x_{0}{ }^{*}[n] y_{0}[n]\right], \quad E\left[x_{1}^{*}[n] y_{1}[n]\right], \cdots E\left[x_{M-1}{ }^{*}[n] y_{M-1}[n]\right]\right]^{T}$, majorizes the subband cross-correlation vector of any other filter bank. Such a filter bank is called a Principal Component Filter Bank.

In general, the cross-covariance sequence is not Hermitian and not positive definite. However, if a Hermitian, positive definite sequence is used to approximate the cross-covariance sequence, then the PCFB would be the optimal solution to the approximated problem. For the case of a two channel canceler, Figure 4 shows the cross-spectral density computed from the measured cross-covariance sequence of simulated data. The jamming-to-noise ratio (JNR) in this case was 50 $\mathrm{dB}$. Figure 5 overlaps a smoothed version of the crossspectral density with the power spectral density of the main channel. The densities were smoothed by multiplying a short segment of the covariance and cross-covariance sequences centered about zero lag with a Blackman window. As is evident, there is little difference between the two densities. Therefore, the power spectral density of the main channel, $S_{y y}(\omega)$, was used to derive the analysis and synthesis filters of the PCFB.

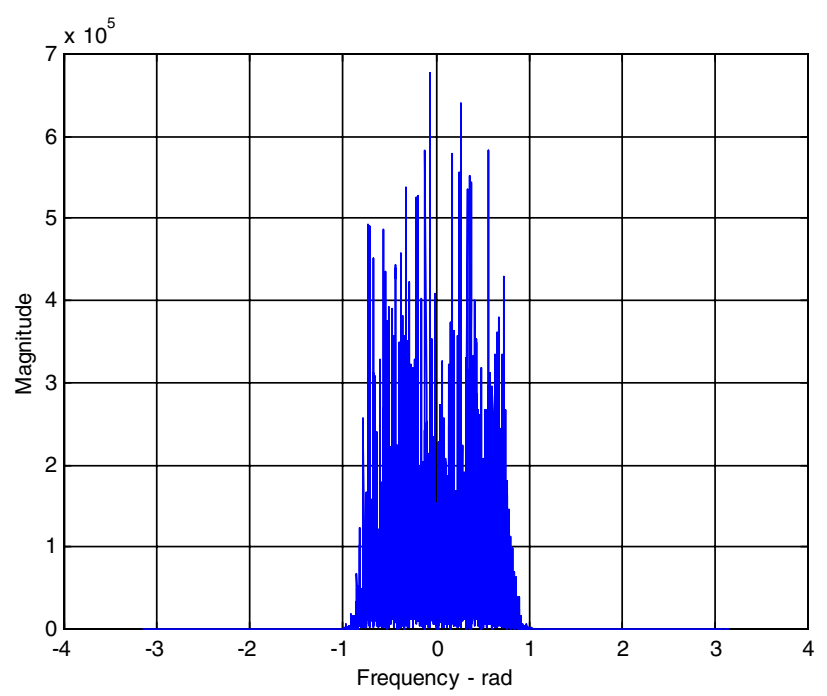

Figure 4. Cross-Spectral Density 


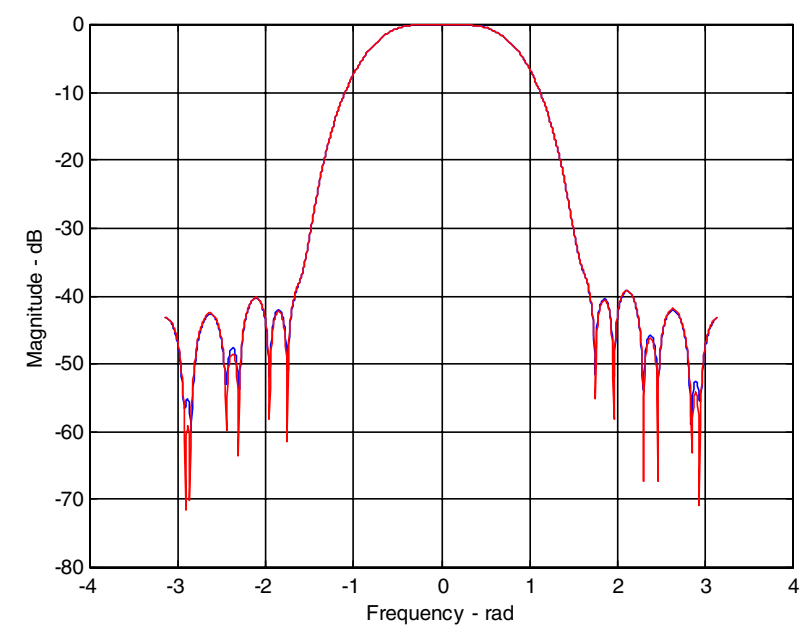

Figure 5. Smoothed Cross-Spectral Density and Substituted Density ( red )

The simulated data for the main and auxiliary channels was generated using the same code originally developed by the authors of References [1] and [2] and generously shared with this author. This signal generation code generates the thermal noise and jamming in the main and auxiliary channels and accounts for the time delay between the two channels. The code allows the user to vary the fractional bandwidth, or the ratio of the signal bandwidth to the center frequency of the array, which is a parameter in this study. Figures 4 and 5 correspond to the case where the fractional bandwidth $f_{b}$ equals 0.5 . Once generated the signals in the main and auxiliary channels are filtered by a 10-pole Butterworth filter with a $3-\mathrm{dB}$ cutoff frequency equal to $\pi / 4$. The filtered signals then proceed as input to the filter bank.

The procedure used for deriving the ideal response of the PCFB analysis filters was taken from Reference [4] and consists of evaluating and ranking the value of the spectral density $S_{y y}(\omega)$ at $M$ equispaced points on the unit circle. If for a given frequency, $\omega, S_{y y}(\omega)$ is the $j$ th largest number in the set

$$
\left\{S_{y y}\left(\omega-\frac{2 \pi k}{M}\right), k=0,1, \ldots, M-1\right\},
$$

then the analysis filters have transfer function

$$
\begin{aligned}
& H_{k}(\omega)=0, \quad k \neq j-1 \\
& H_{j-1}(\omega)=\sqrt{M}
\end{aligned}
$$

for this frequency $\omega$. The resulting filter responses satisfy the Nyquist(M) property, meaning that

$$
\left|H_{i}(\omega)\right|^{2} \downarrow_{M}=1 .
$$

Normally, this procedure yields filters with complicated bandpass structures. Also, the filter responses derived may be very sensitive to changes in the input spectrum. Figures 6 and 7 show the ideal responses of the principal component analysis filters for the case $M=2$. Superimposed on the desired response of the $H_{0}$ and $H_{1}$ filters is the actual response of the designed filter. Figure 8 shows the actual frequency response of the synthesis filters, $F_{0}$ and $F_{1}$.

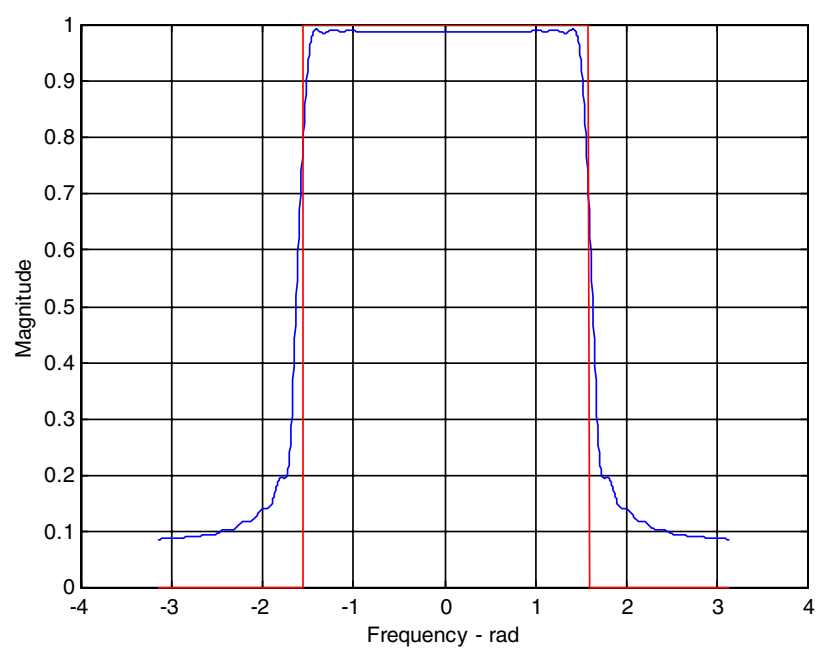

Figure 6. Desired Spectrum ( red ) and $\mathrm{H}_{0}$ Filter Response

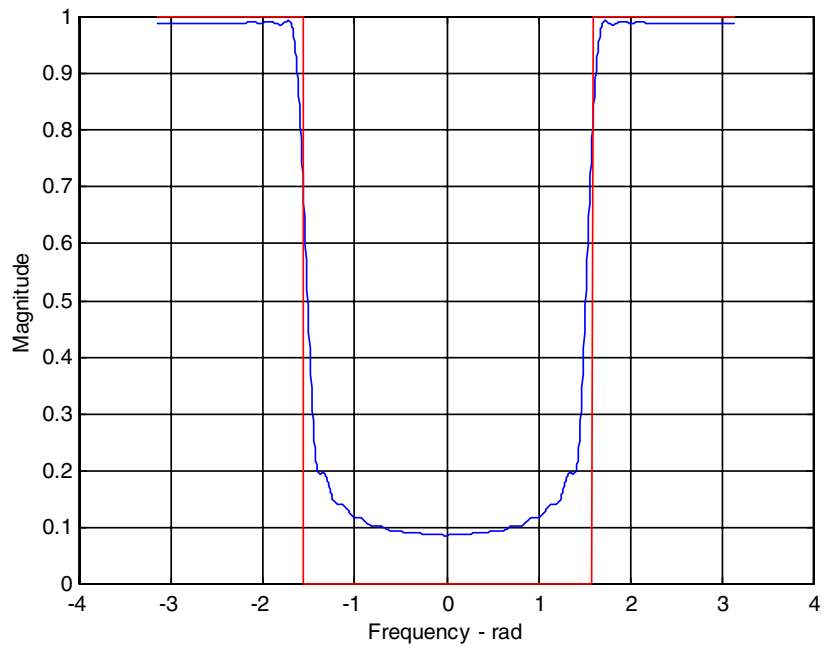

Figure 7. Desired Spectrum ( red ) and $H_{1}$ Filter Response

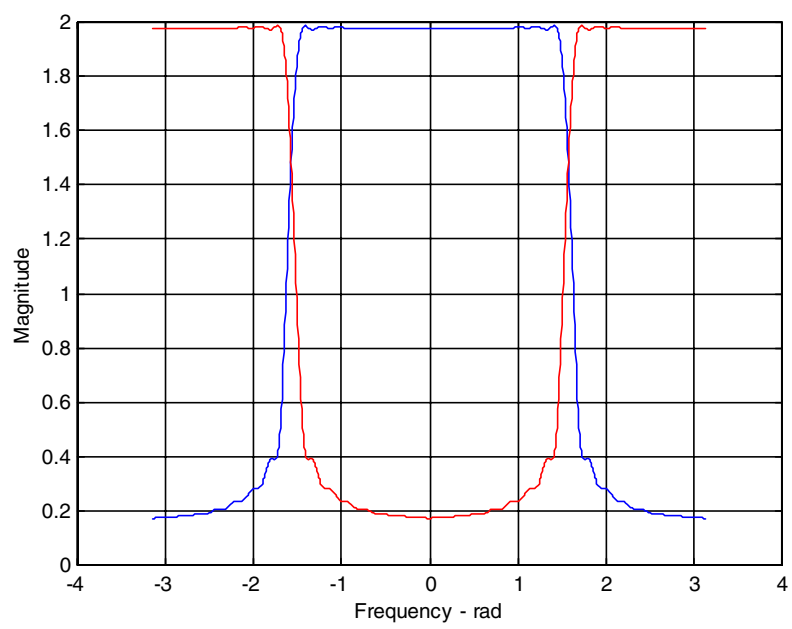

Figure 8. $F_{0}$ and $F_{1}($ red ) Filter Responses 
The filter coefficients were derived using a simple but suboptimal windowing method described in Reference 5. This procedure truncates the impulse response of the magnitude squared desired filter response and multiplies it by a window that has nonnegative frequency response. The spectrum of the windowed sequence will be nonnegative and Nyquist(M). An example of a window with nonnegative frequency response is a triangular window. Next, a spectral factorization procedure yields the coefficients of the $H_{0}$ filter. For a two channel system, the coefficients of the remaining three filters are then uniquely determined. Note that the spectral factors used to create the $H_{0}$ filter are not unique and different spectral factors will yield different filter bank performance as described in Reference [6]. For this study, only the minimum phase and maximum phase solutions were considered. Therefore the zeros of the $H_{0}$ filter were either inside or entirely outside the unit circle. The overall structure of the analysis filter bank is as shown in Figure 9, which results in a uniform frequency domain decomposition.

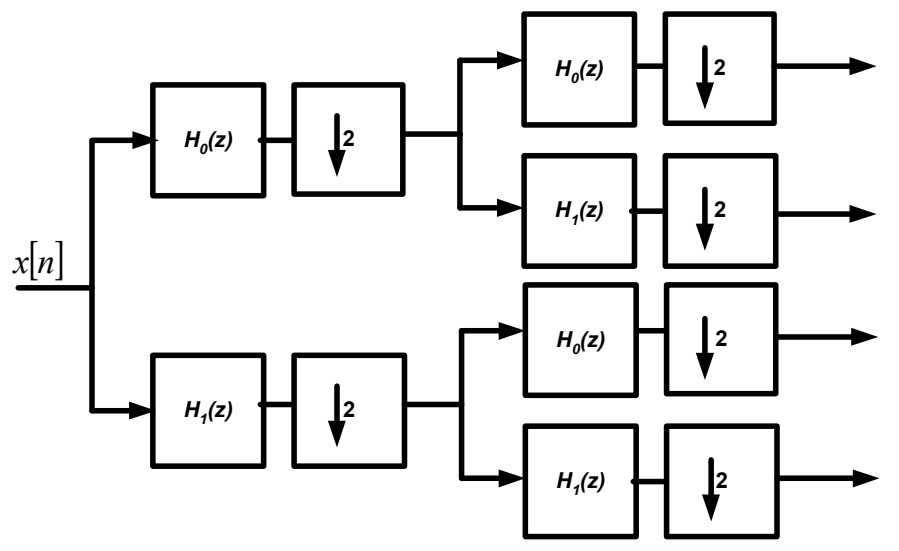

Figure 9. Tree-Structured Analysis Filter Bank

Figure 10 graphs the cancellation performance of the PCFB compared to four other filter banks and a time domain Gram-Schmidt (GS) implementation of the two channel CSLC shown in Figure 1. The other filter banks considered for comparison were a Cosine Modulated Filter Bank, a Dyadic Filter Bank, a Wavelet Packet Filter Bank, and a Discrete Fourier Transform Filter Bank. Each of the filter banks will be described briefly in the following sections.

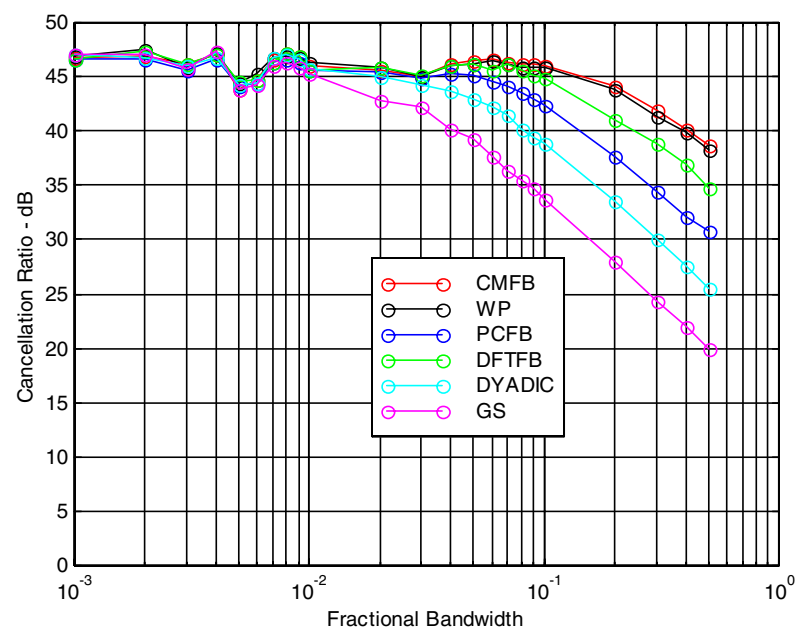

Figure 10. Cancellation Performance

The JNR for all cases shown in Figure 10 was $50 \mathrm{~dB}$ and the fractional bandwidth varied from 0.001 to 0.5 . The CMFB had 32 output channels and the length of the filters was 64 . The WP canceler had 32 output channels and each filter had 32 taps. The DFTFB had 32 channels and to improve performance the input data segments were overlapped by $50 \%$. The PCFB had 32 output channels configured as in Figure 9 and each filter had 32 taps. The maximum phase spectral factor was used to design the $H_{0}$ filter. Finally, the Dyadic canceler had 6 output channels and the length of each filter was 32. All of the filter banks implemented in this study were verified to yield perfect reconstruction.

\section{COSINE MODULATED FILTER BANK}

A CMFB is implemented by designing a prototype lowpass filter and then modulating this filter to cover the entire frequency band. The filter bank is configured as in Figure 3. If the length of the filters, $N$, is a multiple greater than one of the number of filter bank channels, $M$, then the data is processed in overlapping blocks. For example, if $N=2 \mathrm{M}$ as for Figure 10, then the input data is overlapped by $50 \%$. The filter bank is orthogonal since the inverse of the analysis polyphase matrix $E(z)$ is the synthesis polyphase matrix $R(z)$. The impulse response of each modulated filter in the filter bank can be found by using the equation,

$$
h_{k}[n]=h_{0}[n] \sqrt{\frac{2}{M}} \cos \left[\omega_{k}\left(n+\frac{M+1}{2}\right)\right], k=1, \ldots, M-1
$$

where the modulating frequencies are

$$
\omega_{k}=\left(k+\frac{1}{2}\right) \frac{\pi}{M}
$$

and $h_{0}[n]$ is the prototype filter. Figure 11 illustrates the CMFB used for this study. Reference [7] details the theory behind CMFBs. 


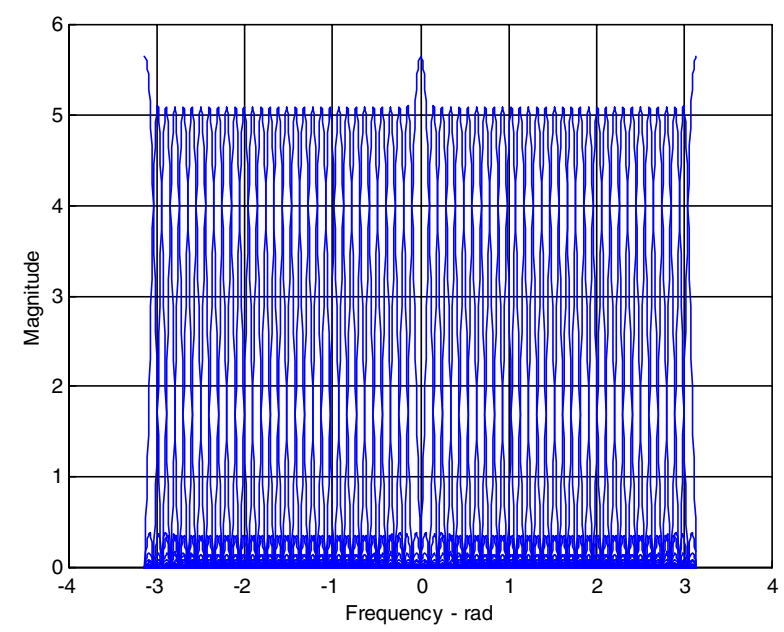

Figure 11. Cosine Modulated Filter Bank

\section{DYADIC FILTER BANK}

A dyadic filter bank iteratively splits the low-pass channel using two analysis filters to create a nonuniform frequency decomposition. The structure of a dyadic filter bank is illustrated in Figure 12. The high-pass channel is pruned at each iteration. The dyadic filter bank produced the worst results of all the filter banks evaluated for this study.

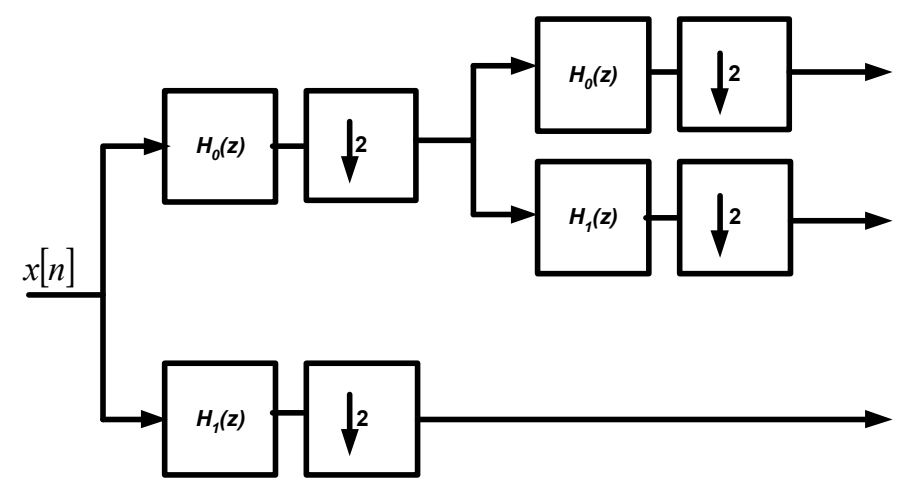

Figure 12. Dyadic Filter Bank

The analysis filters $H_{0}$ and $H_{1}$ used for this particular filter bank are called conjugate quadrature filters and have the property that

$$
\left|H_{0}(\omega)\right|^{2}+\left|H_{1}(\omega)\right|^{2}=1 .
$$

\section{WAVELET PACKET FILTER BANK}

The Wavelet Packet Filter Bank has the same structure as shown in Figure 9 and therefore results in a uniform decomposition of the frequency domain. The analysis and synthesis filters were derived via the spectral factorization of a given half-band filter to yield an orthogonal filter bank. The final filters satisfy the following conditions,

$$
\begin{aligned}
& F_{0}(z)=H_{1}(-z)=z^{-(N-1)} H_{0}\left(z^{-1}\right) \\
& F_{1}(z)=-H_{0}(-z) .
\end{aligned}
$$

\section{DISCRETE FOURIER TRANSFORM FILTER BANK}

The DFTFB has the structure shown in Figure 3 where the polyphase matrices are

$$
E(z)=\bar{F}_{M}, \quad R(z)=F_{M} .
$$

The matrix $F_{M}$ is the DFT matrix and $\bar{F}_{M}$ is its conjugate. To improve cancellation performance the input data to the DFTFB was overlapped by $50 \%$ to yield the results shown in Figure 10.

\section{CONCLUSIONS}

This study investigated the feasibility of applying PCFBs to the problem of subband adaptive cancellation. All the filters yielded nearly equal performance in the narrowband case. For wideband signals, the PCFB did not perform as well as a CMFB, a WP Filter Bank, or a DFTFB, but it did perform better than a Dyadic Filter Bank and the time domain GramSchmidt canceler. The suboptimal performance of the PCFB is attributed to the method used to design the filters, which resulted in filters with high sidelobes.

\section{REFERENCES}

1. F. Lin, K. Gerlach, and M. Picciolo, "BandPartitioned Sidelobe Canceler for a Wideband Radar", Proc. IEEE Radar Conf., Huntsville, AL., May 5-8 2003. 2. P. Cahill, K. Gerlach, and F. Lin, "A Method of Sidelobe Cancellation Using Wavelet Packets", Proc. IEEE Radar Conf., Philadelphia, PA., April 26-29 2004.

3. P. P. Vaidyanathan, Multirate Systems and Filter Banks, Prentice Hall, New Jersey, 1993.

4. M. Tsatsanis, and G. Giannakis, "Principal Component Filter Banks for Optimal Multiresolution Analysis", IEEE Transactions on Signal Processing, Vol. 43, No. 8, August 1995, pp. 1766-1777.

5. A. Kirac, and P. P. Vaidyanathan, "Theory and Design of Optimum FIR Compaction Filters", IEEE Transactions on Signal Processing, Volume 46, No. 4, April 1998, pp. 903-919.

6. A. Tkacenko, and P. P. Vaidyanathan, "On the Spectral Factor Ambiguity of FIR Energy Compaction Filter Banks", IEEE Transactions on Signal Processing, manuscript accepted.

7. H. Malvar, "Extended Lapped Transforms: Properties, Applications and Fast Algorithms", IEEE Transactions on Signal Processing, Volume 40, No. 11, November 1992, pp. 2703-2714. 